\title{
La felicidad de los jóvenes
}

\author{
Namkee Ahn \\ Universidad de Cantabria. Departamento de Economía \\ ahnn@unican.es
}

Francisco Mochón Morcillo

Rebeca de Juan

UNED. Departamento de Análisis Económico II

fmochon@cee.uned.es; rdejuan@cee.uned.es

Recibido: 16-04-2010

Aceptado: 26-07-2011

\section{Resumen}

El bienestar subjetivo de los jóvenes es diferente del de otros colectivos debido a las distintas circunstancias que se dan a lo largo del ciclo vital. Este trabajo contribuye a dar a conocer cuáles son las dimensiones específicas y objetivas del grado subjetivo de satisfacción de los jóvenes españoles. Su finalidad es analizar los factores que inciden en ello.

Los resultados obtenidos indican que los jóvenes casados son más felices que los solteros o los que viven en pareja. El joven que estudia es más feliz que el que trabaja y, dentro del grupo de jóvenes trabajadores, son más infelices los que tienen contratos temporales. La felicidad aumenta con la renta. A diferencia de lo obtenido para el conjunto de la sociedad española, la religión no parece influir en el grado de satisfacción por la vida de los jóvenes. Expectativas positivas aumentan la felicidad de los jóvenes, aunque en menor medida de lo que éstas afectan al conjunto de los españoles. Los jóvenes que viven independientes son más felices que los que viven con sus padres.

Palabras clave: factores demográficos y socioeconómicos; expectativas.

\section{Abstract. Youth happiness}

The subjective well-being of young people is different from other groups due to various circumstances that occur throughout the life cycle. This work contributes to the knowledge of specific and objective dimensions of subjective satisfaction of Spanish youth. Its aim is to analyze the factors affecting such satisfaction.

The results indicate that young married people are happier than single people or those living with a partner. Young students are happier than those that work, and within the group of young workers, those who have temporary contracts are the least happy. Happiness increases with income. Unlike the findings obtained for the whole of Spanish society, religion does not seem to influence the degree of life satisfaction among young people. Positive expectations increase the happiness of youth to a lesser extent than they affect the whole of the Spanish population. Young people who live on their own are happier than those who live with their parents.

Keywords: demographic and socioeconomic factors; expectations. 


\section{Sumario}
Introducción
3. Conclusiones
1. Los datos utilizados y el método
Referencias bibliográficas
de estimación
Anexo A. Las medias de muestra

2. Factores explicativos y resultados para
el bienestar subjetivo de los jóvenes

\section{Introducción}

El bienestar subjetivo de los individuos varía a lo largo del ciclo vital, pues las circunstancias, las aspiraciones, las preferencias, el sistema de valores y las necesidades específicas de cada fase vital son distintas. Es importante tener en cuenta las necesidades y las aspiraciones de un joven de quince años que vive con sus padres y las de una persona de cuarenta años que tiene una familia a su cargo. Aunque esto es algo obvio, no existen encuestas que pregunten a las personas cuáles creen que son las dimensiones subjetivas de su bienestar en cada fase del ciclo vital. Se cuenta, sin embargo, con algunas investigaciones especificas para determinados grupos de edad, como, por ejemplo, una realizada entre personas mayores de sesenta y cinco años (Bowling et al., 2002). Este colectivo valora su calidad de vida en función de los hechos siguientes: si tienen o no acceso a transporte público, si cuentan con unos ingresos suficientes o según sea su estado de salud. Resulta evidente que un joven de veinte años respondería de forma muy distinta si se le preguntara sobre cuáles son los aspectos que más valora de su calidad de vida (Lucas, 2007; Veenhoven, 1991).

La importancia de considerar las transformaciones de las dimensiones subjetivas del bienestar de los individuos a lo largo del ciclo vital también puede intuirse si se tienen en cuenta los resultados comúnmente aceptados por los investigadores cuando se analiza la relación existente entre la edad y el bienestar subjetivo. Esta relación parece tener forma de U (Oswald, 1997; Graham, 2005). Los jóvenes y los mayores son los que tienen las valoraciones más elevadas de bienestar subjetivo, mientras que las valoraciones más bajas de bienestar subjetivo aparecen en las fases medias del ciclo vital, entre treinta y cuarenta años, cuando las responsabilidades familiares y laborales son mayores (Ahn y Mochón, 2010).

A los argumentos formulados, puede añadirse el derivado de tener en cuenta la reciente teoría sobre el referente conceptual de la felicidad (Rojas, 2005). Esta teoría estudia qué referente conceptual de la felicidad tiene una persona en mente cuando formula un juicio sobre su felicidad y establece que la evaluación subjetiva de la vida en su conjunto se ve influenciada por la noción que la persona tiene de lo que es una vida feliz. Se destaca la importancia de la heterogeneidad de los individuos y el hecho de que el referente conceptual no es el mismo para todo el mundo. Lo que para algunos individuos puede resultar muy importante, para otros puede carecer de relevancia. Lógicamente, 
los jóvenes, comparados con las personas de mediana edad o con los mayores, tienen unas circunstancias vitales diferenciadas y su referente conceptual de la felicidad debe ser distinto. La consecuencia de todo ello es la conveniencia de analizar el bienestar subjetivo entre colectivos homogéneos y que tengan un referente conceptual de la felicidad los más parecido posible (Cummins, 1998).

Así pues, es algo aceptado en la literatura, tanto en base a trabajos de naturaleza empírica como teórica, que el bienestar subjetivo de determinados colectivos de individuos, y en especial de los jóvenes, es diferente del de otros colectivos debido a las distintas circunstancias que se dan a lo largo del ciclo vital. El problema es que, a pesar de este reconocimiento, se ha investigado muy poco sobre tales circunstancias y por ello puede resultar conveniente realizar estudios como el que se aborda en este trabajo y así avanzar en el conocimiento de cuáles son las dimensiones específicas y objetivas del bienestar subjetivo de los jóvenes. En este sentido, el objetivo del presente trabajo es analizar los factores que inciden en el bienestar subjetivo de los jóvenes españoles y tratar de determinar las diferencias respecto a las variables que condicionan la felicidad del conjunto de la población española.

Los argumentos anteriormente formulados se pueden ver reforzados desde la óptica de la sociología de la juventud. La juventud, como colectivo social, es una creación relativamente reciente ${ }^{1}$. La sociedad tradicional sólo distinguía al adulto y al niño. El periodo que va de la infancia a la madurez ha existido siempre, pero las más de las veces como edades de vulnerabilidad. Esta vulnerabilidad era una proyección de la infancia, ya que esta sólo existía bajo el amparo de la familia. Desde la óptica de inicios del siglo XXI, la complejidad de las sociedades avanzadas y la competencia entre los individuos legitimada en el conocimiento justifica la tardía incorporación de los jóvenes a la ciudadanía de pleno derecho y lo que es un elemento de vulnerabilidad. En este sentido, el reconocimiento de la juventud como colectivo aparece con características que suponen más una penalización que una ventaja, especialmente en lo tocante a la emancipación, ya que ésta depende de la plena autonomía económica, lo que deberá incidir en la felicidad de este colectivo.

Para estudiar la felicidad de los jóvenes españoles, el trabajo se ha estructurado como sigue. En el siguiente apartado, se describen los datos utilizados y el método de estimación. El segundo epígrafe cumple un doble cometido. Por un lado, justificar la relevancia de las variables escogidas como determinantes de la felicidad, indicando los resultados disponibles, y analizar los resultados obtenidos en este trabajo, señalando las diferencias encontradas con respecto a la literatura disponible. Para cada una de las variables se analizan las razones que pueden explicar que la variable en cuestión sea un determinante de la felicidad y no que la felicidad sea un determinante de la variable escogida. En el último apartado se recogen las conclusiones finales.

1. August Hollinhead, uno de los pioneros de la sociología de la juventud, definía a los jóvenes al filo de la década de los cuarenta como «aquellos que ya no pueden ser considerados como niños, pero que todavía no son adultos» (Allebeck y Rosenmayr, 1979; Brito Lemus, 1998). 


\section{Los datos utilizados y el método de estimación}

Los datos utilizados provienen de los sondeos sobre juventud llevados a cabo por el Centro de Investigaciones Sociológicas (CIS) encargado por el Instituto de la Juventud del Ministerio de Trabajo y Asuntos Sociales. El periodo muestral es de 2002 a 2006. Las entrevistas se realizaron en la primera quincena del mes de marzo de cada año excepto los años 2004 y 2006. En el primer caso, las entrevistas se realizaron entre los días 14 y 19 de octubre y en el segundo, entre el 31 de marzo y el 12 de abril. La muestra utilizada en este trabajo incluye 5.127 individuos con edades comprendidas entre quince y veintinueve años. Los individuos son distintos cada año. Las variables estadísticas descriptivas se presentan en el anexo A.

La variable dependiente es el grado de satisfacción con la vida actual de los individuos, tal como se define en la encuesta del Barómetro del CIS. El grado de satisfacción de la persona toma una de las cuatro categorías siguientes; nada satisfecha, poco satisfecha, bastante satisfecha y muy satisfecha. En el gráfico 1 se presenta la distribución de frecuencias de dicha variable. Como se observa, más del $85 \%$ de los jóvenes encuestados se encuentran bastante o muy satisfechos con su vida actual. La renta del hogar se estructura en diez intervalos en el Barómetro y se ha convertido en cinco intervalos para evitar contar con un número excesivamente reducido de casos en algunos intervalos.

En el análisis empírico, se tienen en cuenta dos categorías de variables, una de carácter general y otra de carácter particular. Las variables de carácter general estudian la incidencia de las variables demográficas y socioeconómicas sobre la felicidad de los jóvenes. En este grupo, las variables incluidas son las siguientes: género (hombre; categoría omitida, mujer), edad, estado civil (casado, con pareja; categoría omitida, soltero), actividad principal (trabaja, trabaja y estudia, parado,

Gráfico 1. Distribución de la variable grado de satisfacción con la vida actual de los jóvenes españoles (en porcentajes)

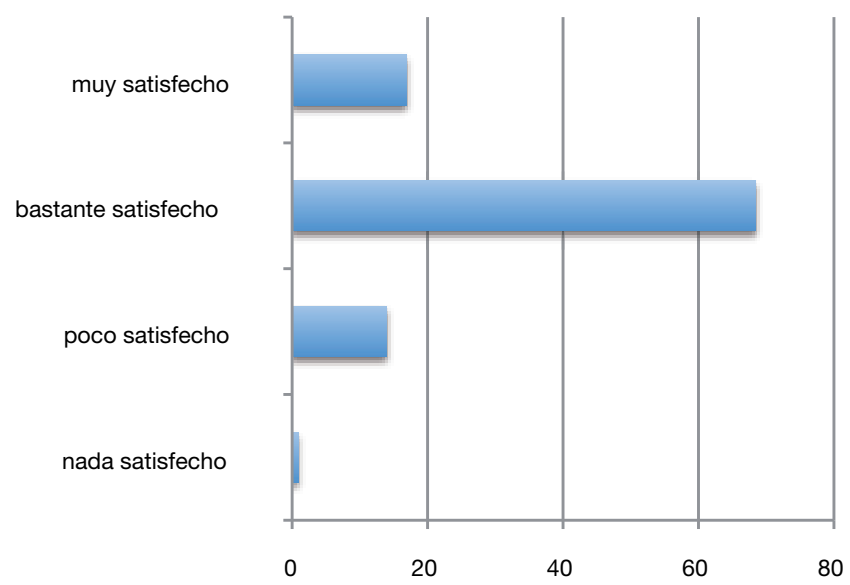

Fuente: elaboración propia. 
labor del hogar; otra situación, categoría omitida, estudia), nivel de renta (cuatro tramos de renta; categoría omitida, nivel de renta malo o muy malo), actitud ante la religión (creyente, practicante; categoría omitida, no creyente).

En la categoría de variables particulares, se incluyen las siguientes: una variable que recoge las expectativas económicas de los jóvenes para el año próximo (mejor o peor), el tipo de escuela en la que se han formado (escuela religiosa, escuela privada; variable omitida, escuela pública) el tipo de contrato laboral que poseen los jóvenes empleados (contrato temporal, empresario, autónomo, otros; variable omitida, temporal) y la situación en la que conviven los jóvenes (viven independientes, comparten vivienda con otros; variable omitida, viven con los padres).

$\mathrm{Al}$ no disponer de información de todas las variables relevantes de cara a la satisfacción vital de los individuos, debe admitirse la posibilidad de sobreestimar los efectos de las variables incluidas por añadir el efecto de otras variables correlacionadas pero no incluidas (un análisis detallado de esta posibilidad se recoge en Argyle, 1999 y Frey y Stutzer, 2002b).

Otra de las limitaciones del análisis se deriva del carácter transversal de los datos utilizados ${ }^{2}$. Los datos transversales no permiten controlar los efectos dinámicos de variables como la adaptación a las nuevas circunstancias (Brickman y Campbell, 1971) o los efectos retardados. Además, es probable que algunas variables explicativas sufran sesgo de endogeneidad. Los remedios para superar esta limitación consisten en utilizar datos longitudinales que controlan efectos fijos individuales o recurrir a variables instrumentales. Dado que en este trabajo no se puede acudir a ninguno de los remedios citados, los resultados deben interpretarse con cautela teniendo en cuenta estos posibles sesgos.

Dada la naturaleza cualitativa y ordinal de la variable dependiente (el grado de satisfacción con la vida), el método de estimación empleado es un probit ordenado. Este método considera sólo los órdenes en el grado de satisfacción, pero no las diferencias cardinales en el citado grado de satisfacción. En este análisis, el nivel de satisfacción toma valores entre 1 (= nada satisfecho) y 4 (= muy satisfecho).

$\mathrm{Al}$ estar formada por jóvenes, podría pensarse que la muestra se trata de un grupo homogéneo, sin embargo, el colectivo investigado es bastante heterogéneo, pues incluye a los jóvenes de quince a veintinueve años ${ }^{3}$. Las circunstan-

2. Los datos de los que se dispone corresponden a individuos diferentes por año, de ahí que tengan que ser considerados en forma de pool de datos para realizar las estimaciones. Sería preferible que se tuviera la información del mismo individuo a lo largo del tiempo, de tal modo que pudiera realizarse un análisis de datos de panel, con las ventajas que este tipo de análisis conlleva.

3. En la sociología de la juventud, definir a la juventud mediante la variable edad ha sido un tema controvertido, ya que la juventud tiene diversas formas de manifestarse y sólo una de ellas es la duración, que es un dato biológico (Bourdier, 1984; Brito Lemus, 1998; Gladys Mathieu, 2005). Sin embargo, para realizar las investigaciones sociológicas, ha sido preciso definir un intervalo de edad. En dichas investigaciones, se ha tendido a una extensión del intervalo considerado, siempre iniciado en quince años de edad, pero que pasa de veinte a veinticinco o a veintinueve años en las investigaciones más recientes (Aguilera, 1994; Gladys Mathieu y Pinto Arboleda, 2010; Mateo Rivas y Navarro López, 1993; Navarro López y Mateo Rivas, 1993; Urraco Solanilla, 2007; Sáez, 1995). 
cias vitales de un joven de quince años, que va a la escuela, vive con sus padres y no realiza ningún trabajo remunerado, tienen muy poco que ver con las de un joven de veintinueve años, casado, que recientemente ha creado un nuevo hogar y que vive del fruto de su trabajo. Las necesidades, las aspiraciones y las preferencias del joven de veintinueve años muy probablemente diferirán mucho de sus propias circunstancias cuando sólo tenía quince años. En el período de tiempo comprendido entre los quince y los veintinueve años, toda persona experimenta unos cambios profundos. Son unos años a lo largo de los cuales el colectivo estudiado estará muy probablemente experimentando una gran transformación, por lo que existirán notables diferencias entre sus integrantes. Durante esos años, se pasa de ir a la escuela a incorporarse al trabajo, de vivir con los padres a hacerlo independientemente, de estar soltero a vivir en pareja, por citar únicamente las transiciones más significativas. Para tener en consideración estas diferencias, el análisis se realiza, en primer lugar, considerando a todos los individuos y, posteriormente, únicamente a los jóvenes que trabajan (cuadro 1). En segundo lugar, se estudia la satisfacción con la vida por grupos de edad, distinguiendo entre quince y diecinueve años, veinte y veinticuatro años y veinticinco y veintinueve años (cuadro 2).

\section{Factores explicativos y resultados para el bienestar subjetivo de los jóvenes}

Los términos felicidad, satisfacción con la vida y bienestar subjetivo, que suelen utilizarse de forma indistinta, en realidad no son sinónimos. La felicidad denota un estado de ánimo. La satisfacción con la vida presupone un juicio consciente que hace la persona sobre su vida o sobre una dimensión de la misma, como el trabajo, las relaciones con los amigos o con la familia. El bienestar subjetivo es un estado personal que integra ambas dimensiones, la emocional y la conscientemente valorativa; es el resultado de cómo las personas perciben y valoran el mundo en el que viven (Ferrer-i-Carbonell, 2002). A pesar de ello, para facilitar la exposición, en este trabajo se utilizan de forma indistinta los tres términos, ya que están íntimamente relacionados.

El bienestar subjetivo depende de la percepción que las personas tienen de su mundo y ésta, a su vez, está condicionada por el temperamento y la personalidad de la propia persona, tema que, si bien tiene una gran importancia, no se considerará en este trabajo. El análisis se va a centrar en los factores que inciden en la percepción que las personas tienen de su vida y se realiza desde la perspectiva de los jóvenes españoles.

Para explicar el nivel de satisfacción de los jóvenes, se ha acudido a una amplia gama de variables explicativas (Layard, 2005). El análisis que seguidamente se presenta se realiza agrupando estas variables en dos grandes categorías. Como se ha señalado, por un lado, se analizan las variables generales y, por otro, se incluyen una serie de variables particulares. La selección de estas variables se ha realizado teniendo en cuenta la información contenida en las encuestas de la base de datos de referencia. 
Cuadro 1. Nivel de satisfacción de los jóvenes españoles

\begin{tabular}{|c|c|c|c|c|}
\hline \multicolumn{5}{|c|}{ Probit ordenado } \\
\hline \multirow[b]{2}{*}{ Variables explicativas } & \multicolumn{2}{|c|}{ Todos } & \multicolumn{2}{|c|}{ Los que trabajan } \\
\hline & Coeficiente & Z & Coeficiente & Z \\
\hline \multicolumn{5}{|l|}{ Variables demográficas y socioeconómicas: } \\
\hline Mujer & 0,035 & 1,01 & 0,024 & 0,43 \\
\hline Edad & 0,047 & 1,00 & 0,021 & 0,20 \\
\hline Edad al cuadrado & $-0,002$ & $-1,52$ & $-0,001$ & $-0,45$ \\
\hline \multicolumn{5}{|l|}{ Estado civil (re: soltero) } \\
\hline Casado & 0,188 & 2,34 & 0,056 & 0,56 \\
\hline Con pareja & $-0,056$ & $-0,67$ & $-0,217$ & $-1,99$ \\
\hline \multicolumn{5}{|l|}{ Actividad principal (re: estudia) } \\
\hline Trabaja & $-0,178$ & $-3,51$ & & \\
\hline Trabaja y estudia & $-0,141$ & $-2,36$ & & \\
\hline Parado & $-0,433$ & $-6,89$ & & \\
\hline Labores del hogar & $-0,137$ & $-1,11$ & & \\
\hline Otra situación & $-0,006$ & $-0,03$ & & \\
\hline \multicolumn{5}{|l|}{ Situación económica (re: mala) } \\
\hline Muy buena & 1,301 & 10,11 & 1,714 & 7,38 \\
\hline Buena & 0,828 & 14,48 & 1,059 & 10,74 \\
\hline Regular & 0,359 & 6,52 & 0,578 & 6,09 \\
\hline \multicolumn{5}{|l|}{ Religión (re: no creyente) } \\
\hline Creyente & 0,032 & 0,82 & $-0,020$ & $-0,32$ \\
\hline Practicante & $-0,031$ & $-0,64$ & $-0,069$ & $-0,85$ \\
\hline \multicolumn{5}{|l|}{ Variables particulares: } \\
\hline \multicolumn{5}{|c|}{ Expectativa económica para el año que viene (re: igual que este año) } \\
\hline Mejor & 0,142 & 3,67 & 0,110 & 1,76 \\
\hline Peor & $-0,147$ & $-3,34$ & $-0,146$ & $-1,98$ \\
\hline \multicolumn{5}{|l|}{ Tipo de escuela (re: publica) } \\
\hline Escuela religiosa & 0,019 & 0,44 & $-0,059$ & $-0,77$ \\
\hline Escuela privada & $-0,012$ & $-0,18$ & $-0,091$ & $-0,81$ \\
\hline \multicolumn{5}{|l|}{ Tipo de contrato si trabaja (re: permanente) } \\
\hline Contrato temporal & & & $-0,193$ & $-3,26$ \\
\hline Empresario & & & $-0,136$ & $-0,65$ \\
\hline Autónomo & & & 0,107 & 1,08 \\
\hline Otros & & & $-0,360$ & $-1,22$ \\
\hline \multicolumn{5}{|l|}{ Convivencia (re: vive con padres) } \\
\hline Vive independiente & 0,209 & 3,09 & 0,257 & 2,91 \\
\hline Comparte con otros & $-0,027$ & $-0,38$ & $-0,069$ & $-0,58$ \\
\hline Número de observaciones & 5.127 & & 1.995 & \\
\hline Pseudos R cuadrado & 0,0626 & & 0,0688 & \\
\hline
\end{tabular}

Fuente: elaboración propia.

Nota: La variable dependiente es el grado de satisfacción con la vida actual. Esta variable toma valor $1 \mathrm{si}$ está nada satisfecho, 2 si está poco satisfecho, 3 bastante satisfecho y 4 muy satisfecho. 
Cuadro 2. Nivel de satisfacción de los jóvenes españoles por tramos de edad

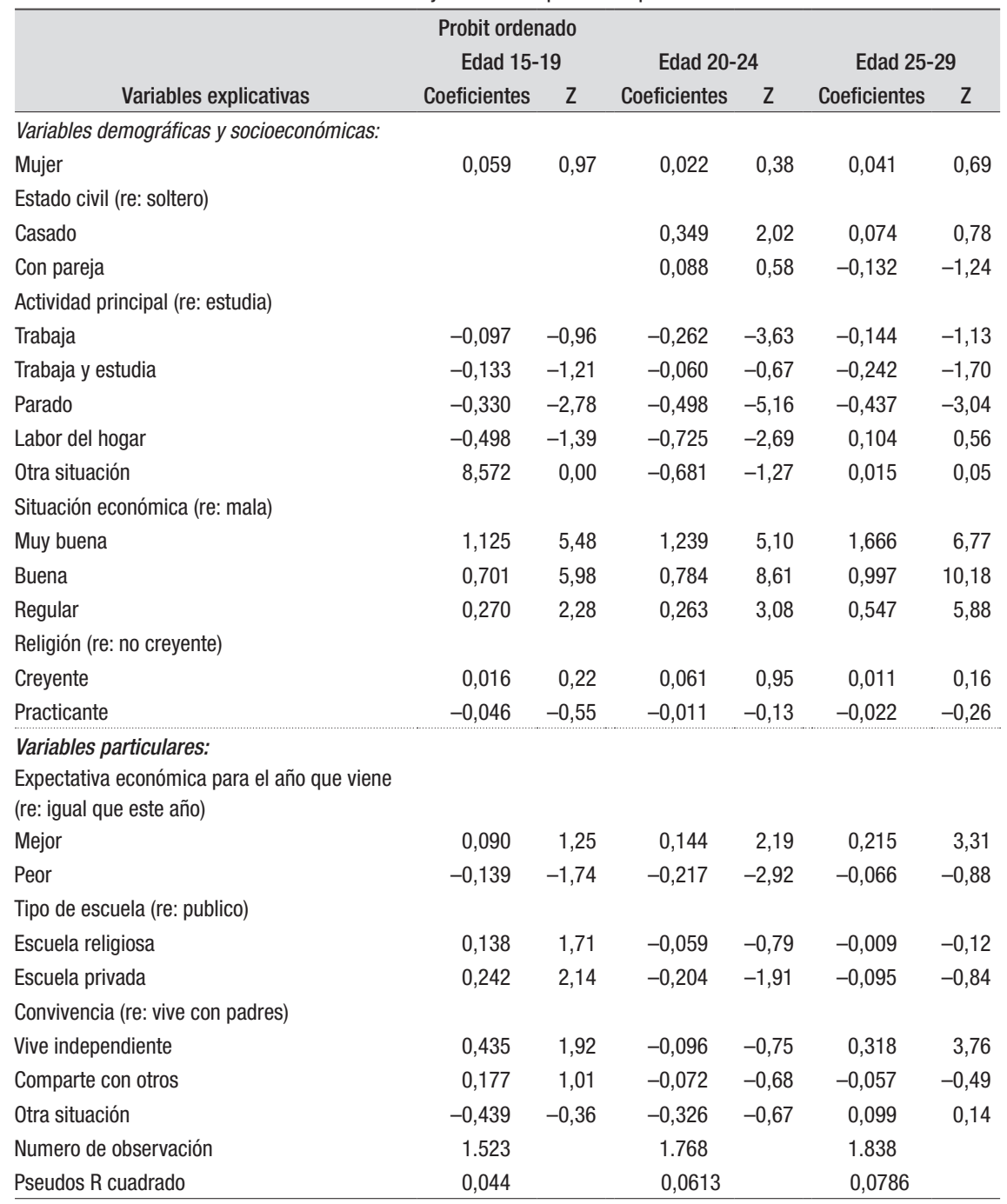

Fuente: elaboración propia.

Nota: La variable dependiente es el grado de satisfacción con la vida actual. Esta variable toma valor $1 \mathrm{si}$ está nada satisfecho, 2 si está poco satisfecho, 3 bastante satisfecho y 4 muy satisfecho.

El procedimiento seguido para presentar las distintas variables y comentar los resultados encontrados para el caso de los jóvenes españoles es el siguiente: en primer lugar, se analiza la relevancia de la variable escogida como determinante de la felicidad; en segundo lugar, se presentan los resultados obtenidos para los jóvenes españoles; por último, se interpreta el resultado, señalando las razones que existen para pensar que la variable 
es un determinante de la felicidad y no que la felicidad es un determinante de la variable escogida. Esta consideración sólo se formula para aquellas variables que resultan convenientes, ya que en otros casos, como la edad o el sexo, no tiene sentido.

\subsection{Variables generales}

En esta primera categoría de variables se analiza fundamentalmente la relación con la felicidad de un amplio número de variables demográficas y socioeconómicas.

\subsubsection{Variables demográficas y socioeconómicas}

\section{Género}

Por lo que respecta a la variable género, la pregunta pertinente es si hay diferencias entre los hombres y las mujeres en relación con su bienestar subjetivo, cuando, objetivamente, su situación es la misma. Según los estudios disponibles, las diferencias entre hombres y mujeres son muy pequeñas (Theodossiou, 1998; Gerdtham y Johannesson, 2001). Aunque las mujeres se deprimen con más frecuencia y experimentan más emociones negativas que los hombres, no se sienten más infelices de forma consistente. Esto se explica porque también experimentan más emociones positivas, de forma que el balance total es neutro. De ello resulta que, de media, hombres y mujeres experimenten un grado de felicidad similar. En un trabajo realizado en Gran Bretaña y Alemania se ha comparado el bienestar subjetivo, en el mismo hogar, del marido y la mujer (Van Praag y Ferrer-i-Carbonell, 2004). Los resultados obtenidos permiten afirmar que las diferencias estructurales entre los hombres y las mujeres con respecto a la satisfacción no son apreciables y, en general, no son estadísticamente significativas.

Los resultados obtenidos para los jóvenes españoles son consistentes con lo señalado, puesto que no se aprecian diferencias debido al género en lo que respecta a la satisfacción subjetiva. Esta variable no resulta ser estadísticamente significativa en ninguna de las estimaciones realizadas, ni considerando toda la muestra, ni por tramos de edad (cuadros 1 y 2).

\section{Edad}

La representación gráfica de la relación entre la edad y la felicidad parece tener forma de U: la felicidad tiende a ser relativamente elevada entre los jóvenes, pues prácticamente no tienen responsabilidades, se reduce conforme aumentan las obligaciones y las complicaciones de la vida, alcanza un mínimo entre los treinta y los cuarenta y cinco años y a partir de entonces empieza a aumentar (Helliwell y Putnam, 2004). Las personas mayores tienden a sentirse más felices, a pesar de que objetivamente suelen estar peor, en el sentido de tener una salud más delicada, de que después de la jubilación su renta se reduce y de que pocos viven aún con su pareja (Argyle, 1999). 
De lo señalado, cabe pensar que los jóvenes como colectivo presentan un nivel de satisfacción con la vida relativamente elevado. Si realmente la relación entre la felicidad y la edad tiene forma de U para el conjunto de los individuos, el colectivo bajo estudio se situaría a lo largo del brazo izquierdo de la U, siguiendo un recorrido descendente hasta aproximarse al mínimo. Pero, dado que, en la muestra analizada, la edad va de los quince a los veintinueve años, sería de esperar que conforme se acerquen a los veintinueve años empezarán a surgir sentimientos contrapuestos. Por un lado, la sensación de autorrealización al conseguir la independencia deberá generar un sentimiento de satisfacción, pero, por otro lado, las obligaciones familiares y laborales también empezarán a aparecer y, con ellas, las tensiones y el estrés. Por ello, en este trabajo, la relación entre felicidad y edad puede que no esté claramente definida para el conjunto del intervalo de quince a veintinueve años.

Los resultados obtenidos en el cuadro 1 indican que la edad no parece que tenga un papel destacado a la hora de explicar la felicidad de los jóvenes españoles. Los coeficientes de las variables de edad presentan los signos adecuados, aunque éstos no son significativos.

\section{Estado civil}

El matrimonio aparece en todos los estudios como uno de los factores más claramente relacionados con la felicidad (Haring-Hidore et al., 1985; Bok, 2010). En general, las personas que están casadas o viven juntas con su pareja son más felices que aquellas que viven solas, bien porque nunca se han casado o porque están separadas, divorciadas o viudas. Además, aquellos que viven junto con su pareja son, por lo general, algo menos felices que los casados y la tasa de ruptura de la pareja es considerablemente superior a la de los casados (Seligman, 2005).

El matrimonio es la mayor fuente de apoyo social para la mayoría de las personas. Más que los amigos o los familiares, la pareja aporta apoyo emocional y material, a la vez que compañía. La pareja desempeña una labor instrumental para una amplia gama de satisfacciones, entre las que cabe incluir sexo, ocio y estabilidad emocional. El matrimonio es bueno para la salud en parte porque propicia una mejor conducta sanitaria, las personas casadas siguen una mejor dieta y suelen cumplir mejor las recomendaciones de los médicos. El matrimonio actúa como una especie de cooperativa en la que cada uno cuida del otro y además mutuamente contribuyen a mejorar su salud mental, ya que cuentan con otra persona con la que intercambiar confidencias y opiniones (Argyle, 1999).

Centrando el análisis en el grupo de los jóvenes (de quince a veintinueve años), cabe indicar que los que estén casados llevarán pocos años y no habrá habido mucho tiempo para que surjan situaciones de divorcio o de viudedad. Por ello, estas dos posibilidades no se consideran. La felicidad de los casados se compara con la de los solteros o los que viven en pareja pero sin haberse casado.

De cara a las hipótesis a contrastar, cabe defender la mayor felicidad relativa de los casados frente a la de los solteros y a los que viven con su pareja sin estar 
casados. Además, los jóvenes casados estarán en los mejores años del matrimonio, cuando las responsabilidades con los hijos y laborales aún son reducidas, por lo cual la felicidad relativa debería ser claramente mayor.

Los resultados obtenidos en este trabajo son, en general, consistentes con lo esperado. Estar casado aparece positivamente relacionado con la felicidad. Esto es así en la estimación realizada con todos los individuos de la muestra (cuadro 1) y para los jóvenes con edades comprendidas entre los veinte y los veinticuatro años. Sin embargo, esta variable no es significativa para los jóvenes de entre veinticinco y veintinueve años (cuadro 2). Este hecho quizás pueda explicarse, como se señala en el estudio de Iglesias de Ussel et al. (2010), por la presencia de hijos en el matrimonio y por la relación de cada cónyuge con la familia política. La llegada de los hijos genera tensiones relacionadas con el reparto desigual de las tareas domésticas y arrebata a la pareja espacios de intimidad. Otro de los factores es una mala relación con la familia política, que puede agudizar las tensiones entre la pareja.

Para plantearse si la relación entre el matrimonio y la felicidad es de causalidad, cabría preguntarse si es más probable que las personas felices se casen. Además del hecho de que aproximadamente el noventa por ciento de la gente se casa, los resultados provenientes de los datos de panel que controlan efectos fijos parecen descartar esta última posibilidad, de forma que la relación de causalidad parece ir desde el matrimonio hacia la felicidad.

\section{Actividad principal}

Para el conjunto de la población, más que de actividad principal se habla de situación laboral. En el caso de los jóvenes, dado que muchos aún no habrán entrado en el mercado laboral, ya que estarán estudiando, debe adoptarse un enfoque más amplio.

Dejando de lado, por el momento, a los jóvenes que solo estudian y centrando el análisis en la situación laboral, son numerosos los estudios que analizan la relación entre la situación laboral y la felicidad (Clark, 2003). De hecho, el paro es una de las variables que más sólidamente está asociada al bienestar subjetivo, pues los desempleados manifiestan una correlación negativa con la felicidad (Kahneman y Krueger, 2006; Clark y Oswald, 1994, Winkelman y Winkelman, 1998; Ahn y Mochón, 2010; Bok, 2010). En prácticamente todos los países, las personas en paro son claramente menos felices que aquellas que tienen empleo. En media, un 11 por ciento de los desempleados se han manifestado como muy felices, mientras que este porcentaje se sitúa en el 30 por ciento para el conjunto de la población (Argyle, 1999). Asimismo, revisando un amplio conjunto de estudios, diversos autores han situado la correlación media entre el empleo y la felicidad en el 0,18 (Argyle, 1999). Cuando se controla el efecto de otros determinantes de la felicidad, tales como la educación y la renta, resulta que la felicidad de los desempleados es bastante menor que la de los empleados con características similares (Frey y Stutzer, 2002b). La pérdida subjetiva de felicidad provocada por el desempleo se ha estimado en 0,33 unidades en una escala de satisfacción que 
va de 1 (no satisfecho en absoluto) a 4 (muy satisfecho). El desempleo incide negativamente en el bienestar más que ninguna otra característica individualmente considerada, incluyendo la separación o el divorcio (Argyle, 1999). La salud mental de los desempleados es peor, con mayores tasas de depresión, suicidio y alcoholismo. Su salud física también es peor y tienen una tasa de mortalidad más elevada.

Estas consideraciones tienen una validez general, sin embargo, pensando en el bienestar subjetivo de los jóvenes, parece oportuno formular ciertas matizaciones. Por un lado, la actividad de estudiante introduce un cierto factor perturbador de la relación entre la situación laboral y la felicidad, no sólo durante los años escolares o universitarios, sino sobre todo en los años de transición al mercado laboral. Una alternativa a permanecer durante unos años trabajando en puestos relativamente mal remunerados y poco considerados, pero necesarios para adquirir experiencia, puede ser invertir en capital humano y ampliar la formación. Las posibilidades son muchas y van desde realizar estudios de postgrado, tipo máster, que permitan una cierta especialización, hasta decidir prolongar los estudios y preparar cualquier tipo de oposición o prueba, necesaria para lograr un trabajo fijo en la Administración o en alguna gran institución. Otra alternativa puede ser optar durante algún tiempo a perfeccionar una lengua extranjera. Por otro lado, la propia consideración de la situación de desempleado se ve influenciada por la edad. El desempleo es una fuente de infelicidad, pues se asocia a la idea de fracaso, sin embargo, este es un concepto relativo. Por ello, cuando en un período de crisis, aumenta mucho el desempleo, los que están en esta situación se sienten relativamente menos mal, pues estar desempleado se considera como algo normal. En este sentido, cabe pensar que entre los jóvenes la situación de desempleado tenga una menor connotación peyorativa que para el conjunto de la población, ya que es un colectivo especialmente afectado por el paro, y porque todo el mundo asume que la incorporación al mercado de trabajo no es algo fácil y requiere un cierto tiempo. El desempleo friccional es algo normal en el funcionamiento del mercado de trabajo y bastante frecuente entre las personas que tratan de incorporarse por primera vez al mismo (Darity y Goldsmith, 1996). Por ello, cuando un joven está en paro, no tiene porque sentirse marginado. Además, los jóvenes no suelen tener responsabilidades familiares, por lo que se ven mucho menos presionados a salir del desempleo de cualquier manera. De hecho, ellos son los se pueden convertir en una carga para la economía familiar.

De cara a las hipótesis a formular, lo primero que debe señalarse es que aquellos que únicamente se dedican a estudiar es de esperar que se sientan felices. Respecto a los que ya han entrado en el mercado de trabajo, cabe esperar que el empleo esté relacionado con la felicidad positivamente, y el desempleo, negativamente; si bien, debido a lo anteriormente comentado, puede esperarse que, entre los jóvenes, la situación de desempleado tenga un menor poder explicativo de la infelicidad que el observado para el conjunto de los individuos.

Para el caso de los jóvenes españoles, los resultados encontrados muestran que la incidencia de la situación laboral sobre la felicidad se ajusta a lo espe- 
rado: Ser estudiante esta positivamente relacionado con la satisfacción. Por el contrario, estar en paro está negativamente relacionado con la felicidad, y esta variable es estadísticamente muy significativa (cuadro 1). La situación de los que trabajan y los que trabajan y estudian también aparece negativamente relacionada con la satisfacción con la vida. Aunque, en un principio, este resultado pueda parecer algo extraño, hay que tener en cuenta que, de cara a su relación con la felicidad, y después de controlar la situación económica y otras variables incluidas en el modelo, los que trabajan, comparados con los que se dedican únicamente a estudiar, están en desventaja. Por tanto, los resultados obtenidos deben interpretarse como que, dada la misma situación en otras variables, los que sólo estudian se sienten más satisfechos que los que trabajan y estudian y los que sólo trabajan. Además, es interesante señalar que este patrón se mantiene cuando se analiza la satisfacción de la vida por grupos de edad (cuadro 2).

¿Es la relación entre empleo y felicidad causal? Una forma de analizar esta posibilidad consiste en hacer estudios longitudinales o de panel entre trabajadores, antes y después de que pierdan su empleo. Diversos estudios han encontrado que la salud de aquellos que han perdido el empleo se ha deteriorado y con ella la felicidad (Argyle, 1999; Ahn et al., 2004). Otros estudios se han realizado entre jóvenes antes de que terminen sus estudios y una vez finalizados, con un intervalo de dos años. Se observa que aquellos que no consiguieron un empleo presentaban una salud mental inferior a los que sí habían encontrado empleo. Ambos tipos de estudios sugieren que la dirección de la causalidad va del empleo a la felicidad.

\section{Renta}

Probablemente, la variable más estudiada en relación con la felicidad sea la renta. En general, los estudios evidencian un efecto positivo de la renta sobre la felicidad. Las personas con niveles más elevados de renta tienen más oportunidades de alcanzar lo que desean y además tienen un estatus social más elevado. De los datos de las encuestas del Eurobarómetro (1975-1991) se desprende que el 88 por ciento del cuartil superior según la renta se mostraban satisfechos o muy satisfechos, mientras que del cuartil inferior el porcentaje de satisfechos era el 66 por ciento (Frey y Stutzer, 2002b). En cualquier caso, probablemente la característica más significativa de la relación existente entre renta y felicidad es que ésta parece ser considerablemente más fuerte para los niveles inferiores de la escala de renta que para los niveles superiores. Esto explica que, a nivel internacional, la relación sea más acusada en los países pobres, como India o Filipinas, que en los ricos. En términos más formales, puede decirse que la relación entre renta y felicidad es no lineal, de forma que el mismo incremento proporcional de la renta produce un incremento inferior de la felicidad para niveles superiores de renta.

Una de las razones por las que una renta más elevada no se traslada de forma directa a una mayor felicidad es porque los individuos se comparan con otros. Esta idea de renta relativa forma parte de la teoría del nivel de ambición o aspiración (Clark et al., 2007). El modelo del gap entre objetivos y logros 
establece que la felicidad se relaciona con la diferencia entre aspiraciones y logros y este gap es debido a comparaciones, tanto con el individuo medio como con el propio pasado del individuo. En base a esta teoría, Easterlin $(1995,2001)$ usa el concepto de las aspiraciones como una referencia para explicar la felicidad. Reconoce que los individuos con rentas más altas son, en promedio, más felices, pero un aumento de la renta de todos los individuos no incrementa la felicidad de todos, pues, comparativamente con los demás, la renta no ha aumentado. Los individuos se comparan con otros y no suelen usar juicios absolutos. En este sentido, es importante saber con qué otros individuos se realizarán tales comparaciones. En un estudio entre los trabajadores británicos, se encontró que cuanto menor sea la renta del grupo de referencia con el que se comparan los entrevistados, más satisfechos se sentirán (Argyle, 1999). Así pues, es la renta relativa más que la renta efectiva la que, a partir de un cierto nivel, hace más felices a los individuos.

Centrándose en la relación que se espera que exista entre la renta y la felicidad de los jóvenes, una primera consideración a formular es que, para muchos jóvenes, para todos aquellos que no se han independizado, la renta en realidad es la renta de los padres. La transición a una renta propia puede que no sea algo completamente radical, sino que, durante un cierto tiempo, se superponga la renta generada por el joven y la que le faciliten sus padres a modo de ayuda en múltiples formas. En el caso de que la renta del joven en realidad sea una renta familiar, es probable que relativamente se valore menos que si fuese autogenerada. Se suele dar una cierta tendencia a no valorar en su justa medida aquello que nos viene dado. Por ello, en el caso de los jóvenes que aún no se han independizado, es lógico pensar que a la renta le otorguen menos importancia que los jóvenes que viven de su propio trabajo.

De esta consideración se infiere, de cara a la contrastación empírica realizada en este trabajo, que parece lógico esperar que los jóvenes de menor edad le otorguen menos importancia a la renta que los de mayor edad. Lo normal será que los jóvenes menores de diecinueve años vivan con sus padres, dependan económicamente de ellos y, consecuentemente, valoren relativamente menos la renta como factor explicativo de su felicidad. Por el contrario, lo normal será que los jóvenes entre veinticinco y veintinueve años se hayan independizado económicamente, estén empezando a crear una familia y valoren bastante la renta como factor que contribuye a su bienestar subjetivo. Además, estos jóvenes asociarán sus logros económicos como una muestra del éxito personal en la vida.

Los resultados encontrados en este trabajo indican que la satisfacción de los jóvenes aparece positivamente correlacionada con el nivel de renta (cuadro 1). Los resultados son consistentes con lo que se viene indicando y muestran que la capacidad económica tiene una gran importancia para los jóvenes. Tener una situación económica muy buena, buena o regular se manifiestan como las variables que están más claramente asociadas con la felicidad de los jóvenes. El coeficiente de los que manifiestan tener una muy buena situación económica es estadísticamente muy significativo. Estos resultados sugieren que la renta 
es muy importante para los jóvenes, ya que se asocia con el hecho de haber tenido, a una temprana edad, la capacidad para triunfar en la vida. Además, como cabía esperar, la incidencia sobre la satisfacción con la vida de la situación económica crece con la edad (cuadro 2).

Tratando de establecer alguna evidencia de causalidad, se ha comparado la felicidad de un grupo de familias en las que el cabeza de familia había experimentado un aumento en sus salarios durante el año anterior con otro grupo de familias que habían sufrido una disminución de los salarios. Los resultados mostraron que las familias que habían experimentado un aumento tenían unos niveles de felicidad mayores (Argyle, 1999). Asimismo, Inglehart (1990) encontró que el 85 por ciento de aquellos que afirmaban que su situación financiera era «mucho mejor» se manifestaban satisfechos, mientras que de aquellos que decían que su situación era «mucho peor» sólo se mostraban satisfechos un 57 por ciento.

Otro tipo de estudios son los realizados entre personas a las que les ha tocado la lotería o que han recibido una herencia (Frey y Stutzer, 2002a). En ambos casos, los individuos manifiestan que su bienestar subjetivo en el año siguiente ha aumentado, lo que de nuevo viene a sugerir que la causalidad va desde la renta hacia la felicidad. A más largo plazo, sin embargo, los efectos sobre la felicidad son poco significativos, lo que se puede explicar por la teoría de la adaptación, según la cual los individuos reaccionan ante un evento positivo o negativo, pero con el transcurso del tiempo vuelven a su nivel habitual de satisfacción (Brickman y Campbell, 1971; Myers, 1992; Lucas et al., 2004). Además, habría que añadir que, según señala el profesor Seligman, todas las personas, en su fórmula individual de felicidad, tienen un componente o rango fijo que no depende de las circunstancias ni de su voluntad (Seligman, 2005). Este rango tiene mucho que ver con factores genéticos y biológicos y difiere notablemente de unos individuos a otros.

\section{Religión}

En muchos estudios empíricos se ha evidenciado que el efecto sobre la felicidad de tener creencias religiosas es positivo, siendo éste aún mayor para el caso de los practicantes y para las personas mayores (Inglehart, 1990; Bok, 2010). Así, el 84 por ciento de aquellos que van a la iglesia con asiduidad se declaran muy satisfechos con su vida, mientras que entre los que nunca van a misa dicho porcentaje es del 77 por ciento (Inglehart, 1990).

Cuando se aplican controles para las variables demográficas —edad, sexo o clase social-, el efecto de la religión sobre la felicidad se reduce, pero sigue siendo apreciable, próximo al 0,15 . Si los controles se extienden a las variables sociales, el coeficiente continúa reduciéndose y se sitúa próximo a 0,10 (Argyle, 1999).

Si la religión afecta a la felicidad, ¿cuál es la explicación? El factor principal parece ser el fuerte apoyo social que las iglesias ofrecen a sus miembros. Por ello, algunos autores señalan que, más que un tema de creer en algo, se trata de pertenecer y participar en una comunidad que apoya y da consuelo a sus 
miembros. La religión con sus ritos propicia compartir experiencias emotivas y sentirse miembro de una comunidad en la que el lazo de unión es una fe común. Para los creyentes, es importante sentir que se participa en una comunidad sagrada. Este tipo de hechos contribuyen a propiciar el sentimiento de que se está cerca de Dios, y esta relación, al igual que ocurre con las relaciones humanas, incide positivamente en la felicidad (Argyle, 1999). Asimismo, tener firmes creencias y certezas existenciales afecta a la felicidad, independientemente de la asistencia a la iglesia. Estos sentimientos incluyen creer en la otra vida, lo que es muy importante especialmente para los ancianos, y en un Dios generoso y compasivo que se preocupa de nosotros.

Centrándose en la investigación objeto de este estudio, la cuestión es si los comentarios formulados tienen validez para el caso de los jóvenes. En un principio, cabe pensar que los jóvenes son menos proclives a plantearse preguntas trascendentales. Además, en la juventud se tiende a valorar menos el soporte que la religión ofrece para hacer más aceptables las adversidades. Por ello, cabe esperar que, para los jóvenes, la religión como factor explicativo de la felicidad tenga una importancia relativa inferior a la observada para el conjunto de la sociedad.

Los resultados encontrados en esta investigación son consistentes con lo esperado. Para los jóvenes españoles, no existe una relación significativa entre religión y felicidad (cuadro 1). Este hecho permite afirmar que, en contra de lo observado en el conjunto de la sociedad española (Ahn y Mochón, 2010), para los jóvenes la religión no incide en su satisfacción por la vida. Este resultado se mantiene al realizar el análisis por tramos de edad (cuadro 2).

Para analizar la posible relación de causalidad entre religión y felicidad, se han llevado a cabo diversos estudios. Unos han permitido predecir la mayor felicidad relativa de aquellos grupos de personas mayores que habían estado asistiendo con asiduidad a la iglesia durante una serie de años. Otros han evidenciado que las experiencias religiosas producen un efecto estimulante sobre la felicidad de aquellos que las han vivido, y las conversiones religiosas también tienen efectos beneficiosos sobre la felicidad (Argyle, 1999). Todo ello sugiere que la relación de causalidad va de la religión a la felicidad.

\subsection{Variables particulares}

\section{Efecto del pasado, del presente, del futuro: las expectativas}

Uno de los procesos más importantes que los individuos deben experimentar es ajustarse a experiencias del pasado. Los seres humanos o bien son incapaces o no les gusta realizar juicios absolutos. Prefieren estar constantemente realizando comparaciones con el pasado o con las expectativas sobre su futuro (Frey y Stutzer, 2002b). Esto implica que la satisfacción presente de los individuos, además de su situación actual, dependa de sus experiencias pasadas y de lo que se espera para el futuro (Van Praag y Ferrer-i-Carbonell, 2004).

Aunque el proceso por el que la memoria actúa incidiendo en el presente está aún en estudio (Kahneman et al., 1999), parece lógico pensar que la satis- 
facción que producirá un acontecimiento determinado ejercerá una menor incidencia si este ocurrió hace un mes que si ocurrió ayer. En cualquier caso, estudios recientes permiten afirmar que se puede estimar el efecto sobre la felicidad de acontecimientos ocurridos a lo largo del horizonte temporal y que no parece existir una pérdida de continuidad entre los recuerdos acontecidos en el pasado y las expectativas que se forman sobre acontecimientos futuros (Loewestein, 1987; Van Praag y Ferrer-i-Carbonell, 2004).

Por lo que respecta al papel de las expectativas, el hecho es que, cada vez más, la gente se preocupa del futuro. Las expectativas sobre lo que será nuestra vida forman parte de nosotros e inciden sobre la felicidad. Cuando las expectativas son favorables, nos sentimos felices, pero cuando, por algún motivo, ese sentido de futuro se resquebraja, nos sentimos mal y vulnerables. La incertidumbre nos preocupa porque se nos rompe el esquema sobre cómo teníamos pensado que discurriría nuestra vida (Senik, 2008; Ahn y Mochón, 2007).

De cara a la presente investigación, la pregunta pertinente es en qué medida los jóvenes se sentirán sensibilizados por su futuro. En un principio, parece lógico pensar que a toda persona, cualquiera que sea su edad, le preocupa qué es lo que le deparará el futuro. Admitiendo este hecho, el siguiente paso es si los jóvenes, dadas sus circunstancias vitales, se sentirán más o menos preocupados por su futuro que los adultos. En este sentido, aunque es un tema a investigar, cabría pensar que los jóvenes pueden estar relativamente más inclinados a vivir el presente y preocuparse menos por las expectativas sobre su futuro. Aunque esta actitud no es privativa de los jóvenes, sí puede ser más frecuente entre ellos (Rojas, 2005). Además, y pensando exclusivamente a título de ejemplo en un referente ligado al ahorro, lo normal es que las personas sólo empiecen a ahorrar para sus planes de pensiones a partir de una cierta edad. Esto ocurrirá cuando el nivel de ingresos sea suficiente para cubrir sus necesidades inmediatas y puedan empezar a pensar en el futuro.

La contrastación empírica de este tipo de temas requiere una información a la que no siempre resulta posible acceder. Por lo que respecta a las expectativas, es lógico pensar que éstas, en cierto modo, están incorporadas en la situación actual, por lo que, en realidad, la información disponible no es puramente sobre expectativas. En cualquier caso, los individuos manifiestan claramente su opinión sobre la evolución futura y es especialmente relevante la incidencia de las expectativas económicas sobre el nivel de vida.

Los resultados obtenidos en este trabajo son consistentes con lo esperado y el impacto de las expectativas globalmente consideradas se ajusta a las hipótesis previamente formuladas: unas expectativas favorables aparecen positivamente correlacionadas con la satisfacción de los individuos con su nivel de vida, mientras que si las expectativas son desfavorables, la correlación es negativa (cuadro 1). Además, la importancia de las expectativas sobre el futuro entre los jóvenes españoles es otro factor que varía con la edad. Los jóvenes de veinticinco a veintinueve años valoran más el futuro que los de quince a diecinueve, lo que puede interpretarse como un signo de madurez (cuadro 2). 
La relación de causalidad entre las expectativas y la felicidad parece ir, en un principio, desde las expectativas hacia la felicidad, ya que cuando una persona tiene unas expectativas de futuro favorables, esto le hace sentir más feliz. En cualquier caso, también cabe argumentar que aquellas personas que están felices tienden a ver el futuro de forma más positiva que aquellas otras que están atravesando una mala temporada y se muestran insatisfechas.

\section{Tipo de enseñanza recibida}

Los estudios comparados muestran una estrecha relación entre los valores culturales y el bienestar de las personas. En este sentido, cabe la posibilidad de que, según el tipo de enseñanza recibida, se hayan podido transmitir unos valores distintos. Como señala Sandoval Manríquez (2007: 117): «en el marco de la escuela como institución se da una interacción constante entre la estructura, la organización y la metodología didáctica. Estos conllevan a juicios de valor y convierten a estos medios en vehículos decisivos de esquemas de valoración y de adhesión a determinados valores». Si esto fuese así, sería conveniente analizar su impacto sobre la felicidad. En esta investigación, las posibilidades consideradas, según el tipo de enseñanza recibida, son: haber asistido a un colegio público, a un colegio privado religioso o a un colegio privado laico.

Respecto a los posibles resultados de la contrastación, es difícil formular claramente hipótesis muy definidas. Por un lado, la asistencia a un colegio religioso podría ser un indicador de una mayor religiosidad, lo que, en base a lo señalado, al estudiar la religión como factor explicativo de la felicidad, podría estar relacionado con unos mayores niveles de bienestar subjetivo. Por otro lado, cabría esperar que, en los centros de enseñanza públicos o privados, pero laicos, se imparta una enseñanza en la que se inculquen valores más individualistas, donde el bienestar de grupos primarios como la familia ocuparía un segundo plano. Este entorno más liberal e individualista ofrecería un mayor estímulo para la realización personal. Esto podría explicar que, en los centros laicos, más individuos estén muy satisfechos con su vida (Diener et al., 1997).

Los resultados obtenidos sugieren que haber asistido a un colegio público, a un colegio religioso o a un colegio privado laico no supone un hecho diferencial sobre la felicidad (cuadro 1). La relación no es significativa en ninguna circunstancia. En cualquier caso, al realizar el análisis por tramos de edad, se observa que el tipo de escuela en el que se han formado los jóvenes influye positivamente sobre la felicidad en el grupo de los de menor edad (de quince a diecinueve años) (cuadro 2).

\section{El tipo de contrato laboral}

La importancia de la situación laboral de cara a la felicidad ha sido señalada por otros investigadores (Ferrer-i-Carbonell y Van Praag, 2006). La diferencia entre estar empleado o desempleado representa un abismo de cara a la felicidad de los individuos, como se ha indicado al analizar la variable actividad principal, pero no todas las situaciones de los empleados son las mismas. Al margen de las diferencias asociadas al puesto de trabajo, hay otras que se derivan del tipo 
de contrato. Resulta lógico pensar que los contratos eventuales, algo que está muy extendido entre los jóvenes, conlleven un componente de incertidumbre y provisionalidad que los diferencie de los contratos indefinidos. Por ello cabría esperar que los contratos eventuales estén asociados negativamente con la felicidad.

Los resultados son consistentes con las hipótesis formuladas y permiten afirmar que tener un contrato eventual, en contraposición a un contrato permanente, incide negativamente sobre la felicidad, siendo esta variable claramente significativa.

\section{Situación respecto a la vivienda}

Las variables hasta ahora analizadas son las que suelen incluirse en los estudios sobre el bienestar subjetivo de los individuos. En el caso de la encuesta sobre los jóvenes que se ha tomado como base para realizar esta investigación, se ha incluido esta variable que está específicamente pensada para los jóvenes. Se pregunta a los jóvenes si viven con sus padres, si se han independizado y viven solos, si comparten vivienda con otros jóvenes o si recurren a otras soluciones para resolver su problema de vivienda. La relación con la felicidad de la forma en que los jóvenes resuelven su problema de vivienda puede ser importante y, sin duda, tiene relevantes implicaciones. Las circunstancias de un joven que vive con sus padres son muy distintas de las de aquel que ha tenido la capacidad para independizarse (Iglesias de Ussel et al., 2010). Poder abordar el coste económico que conlleva mantener una vivienda supone una capacidad importante, no sólo financiera, por parte de los jóvenes. Por ello, cabría esperar encontrar unos valores relativamente elevados de satisfacción con la vida entre aquellos que se han independizado en términos de vivienda.

Los resultados encontrados corroboran esta hipótesis, pues indican que aquellos jóvenes que viven independientes de sus padres son los que presentan un mayor grado de satisfacción (cuadro 1). Una posible justificación de este hecho es que cuando un joven puede independizarse de sus padres y vivir por sí mismo, esto implica que tiene suficiente capacidad económica para hacerlo. Tener esa capacidad supone un logro importante a esa edad y un motivo de satisfacción. Este patrón se repite cuando se realiza el análisis por tramos de edad. El efecto positivo sobre la felicidad de vivir de forma independiente es más significativo para el grupo de más edad (de veinticinco a veintinueve años) (cuadro 2).

\section{Conclusiones}

El bienestar subjetivo de colectivos específicos de individuos, como es el caso de los jóvenes, es diferente del de otros colectivos debido a las distintas circunstancias que se dan a lo largo del ciclo vital. A pesar de este reconocimiento, se ha investigado muy poco sobre tales diferencias. Este trabajo pretende contribuir al conocimiento de cuáles son las dimensiones específicas y objetivas del bienestar subjetivo de los jóvenes españoles. 
Para llevar a cabo este análisis, las posibles variables explicativas se han agrupado en dos categorías: variables generales, que son demográficas y socioeconómicas, y variables particulares, como las relacionadas con el pasado, el presente, el futuro, el tipo de enseñanza recibida, el tipo de contrato laboral, etc. La base de datos utilizada es el Barómetro del CIS correspondiente al período 2002-2006.

Antes de presentar un balance final de los resultados obtenidos, debe recordarse que, al haber tenido que limitar el estudio a la información disponible en la encuesta empleada, no se han podido incluir todas las variables relevantes de cara a la satisfacción vital de los individuos. Por ello no se puede descartar la posibilidad de sobreestimación de los efectos de las variables incluidas, debido a que se añade el efecto de otras variables correlacionadas pero no incluidas. Tampoco puede olvidarse que, al utilizar datos de carácter transversal, no se pueden controlar los efectos dinámicos de variables como la adaptación a las nuevas circunstancias o los efectos retardados. Además, es probable que algunas variables explicativas sufran del sesgo de endogeneidad, y es especialmente posible que aparezca este sesgo en el caso de la variable que denota las expectativas.

Por lo que respecta a las variables demográficas y socioeconómicas, los resultados más significativos son los siguientes: 1) No se observan diferencias entre los jóvenes españoles según sexo en lo que respecta al bienestar subjetivo. 2) La edad tampoco parece que tenga un papel destacado a la hora de tratar de explicar la felicidad de los jóvenes. 3) Los casados se muestran más felices que los solteros y los que viven con su pareja sin estar casados. Por tramos de edad, la incidencia positiva de estar casado sólo se observa para los jóvenes con edades comprendidas entre veinte y veinticuatro años, mientras que este efecto no se obtiene para los jóvenes de entre veinticinco y veintinueve años. La explicación parece estar en la llegada de los hijos y en la relación de cada cónyuge con la familia política (Iglesias de Ussel et al., 2010). 4) La felicidad aumenta significativamente con la renta. Tener una situación económica mala o muy mala es una causa de insatisfacción, mientras que, por el contrario, contar con una situación económica muy buena, buena o regular se manifiestan como las variables que están más claramente asociadas con la felicidad de los jóvenes. En concreto, la incidencia sobre la satisfacción con la vida de la situación económica crece con la edad. 5) La religión no parece incidir sobre la felicidad de los jóvenes españoles, lo que contrasta con lo que sucede para el conjunto de la población.

El análisis del efecto sobre la felicidad de las variables particulares se inicia con el estudio de las expectativas. En este sentido, se observa que la inclusión de las expectativas como variable explicativa de la felicidad también genera unos resultados consistentes con las hipótesis generalmente aceptadas. Cuando las expectativas genéricamente consideradas son favorables, se correlacionan positivamente con la satisfacción de los individuos, mientras que si las expectativas son desfavorables, la correlación es negativa. Los resultados sugieren, además, que la importancia de las expectativas sobre el futuro entre los jóvenes españoles varía con la edad. 
En general, los resultados del análisis del efecto sobre la felicidad del tipo de enseñanza recibida no son estadísticamente significativos. Únicamente para el grupo de los jóvenes de menor edad se observa que la incidencia del tipo de escuela sea relevante.

$\mathrm{Al}$ analizar la posible incidencia del tipo de contrato en los jóvenes trabajadores, los resultados permiten afirmar que tener un contrato eventual, en contraposición a un contrato permanente, incide negativamente sobre la felicidad. Esta variable es claramente significativa.

El análisis de la incidencia sobre la felicidad de que los jóvenes vivan con los padres, que se hayan independizado, que compartan vivienda con otros jóvenes o que recurran a otras modalidades para resolver sus problemas de vivienda muestra resultados relevantes. Aquellos jóvenes que viven independientemente son los que presentan un mayor grado de satisfacción, además, el efecto es muy significativo. Parece ser que, cuando un joven puede independizarse de sus padres y vivir por sí mismo, esto de por sí supone un logro importante a esa edad y un motivo de satisfacción. Esta idea se ha ratificado al analizar la incidencia de la situación económica sobre la felicidad. Además, el efecto positivo sobre la felicidad de vivir independientemente es mayor para el grupo de jóvenes de más edad.

El análisis realizado sobre la felicidad de los jóvenes españoles debería considerarse como un punto de partida para nuevas investigaciones que traten de profundizar en el conocimiento de los factores que inciden en la satisfacción subjetiva de este colectivo. En concreto, las especiales características del mercado de trabajo en España acentúan la situación de inseguridad y precariedad de los jóvenes, lo que está contribuyendo a alargar ficticiamente la etapa de juventud. Al margen de otras consideraciones, la dualidad existente en el mercado de trabajo español supone una injusticia intergeneracional que, al menos, merecería ser estudiada con cierto detenimiento.

\section{Referencias bibliográficas}

Aguilera, M.J. (1994). «La juventud en España». Espacio, Tiempo y Forma. Serie VII, Geografía, 7, 11-19.

AHn, N.; GARCíA, J.R. y JimENO, J.F. (2004). Well-being consequences of unemployment in Europe. Documento de trabajo, 2004-16. Fedea.

Ahn, N. y Mochón, F (2007). Expectations and happiness. Documento de trabajo, 2007-01. Fedea.

- (2010). «La felicidad de los españoles: Factores explicativos». Revista de Economía Aplicada, XVIII, 54, 5-31.

Allebeck, K. y Rosenmayr, L. (1979). Introducción a la sociología de la juventud. Buenos Aires: Kapelusz.

Argyle, M. (1999). "Causes and correlates of happiness». En: Kahneman, D.; DiENER, E. y SCHWARZ, N. (eds.). Well-being: The foundations of hedonic psychology. Nueva York: Russell Sage Foundation, 353-373.

BоK, D. (2010). The politics of happiness: What government can learn from the new research on well-being. Princeton: Princeton University Press.

Bourdier, P. (1984). Cuestiones de Sociologia. Madrid. ISTMO, 2000. 
Bowling, A.; Gabriel, Z.; Banister, D. y Sutton, S. (2002). Adding quality to quantity: Older people's views on their quality of life and its enhancement [en línea]. Research Findings, 7. Growing Older Programme. <http://www.shef.ac.uk/uni/ projects/gop/index.htm>.

Brickman, P. y CAMPBell, D.T. (1971). "Hedonic relativism and planning the good society». En: Hapley, M.H. (ed.). Adaptation level theory. Nueva York: Academic Press, 287-305.

BRito LEMUS, R. (1998). «Hacia una sociología de la juventud: Algunos elementos para la deconstrucción de un nuevo paradigma de la juventud». Última década, 9.

Clark, A. (2003). "Unemployment as a social norm: psychological evidence from panel data». Journal of Labor Economics, 21, 323-350.

Clark, A.E.; Frijters, P. y SHIELdS, M.A. (2007). Relative income, happiness and utility: An explanation for the Easterlin paradox and other puzzles. Documento de debate, 2840. IZA.

Clark, A.E. y Oswald, A. (1994). «Unhappiness and unemployment». Economic Journal, 104, 648-659.

Cummins, R. A. (1998). "The second approximation to an internacional standard for life satisfaction». Social Indicators Research, 43, 307-334.

Darity, W. y Goldsmith, A.H. (1996). «Social Psychology, unemployment and macroeconomics». Journal of Economic Perspectives, 10, 121-140.

Diener, E.; SuH, E y Oishi, A. (1997). «Recent findings on subjective well-being». Indian Journal of Clinical Psychology, marzo.

EASTERLIN, R.A. (1995). "Will raising the incomes of all increase the happiness of all?». Journal of Economic Behavior and Organization, 27, 35-47. Recent Findings on Subjective WellBeing.

- (2001). "Income and happiness: Towards a unified theory». The Economic Journal, 111, 465-84. Recent Findings on Subjective WellBeing.

FERRER-I-CARBONELL, A. (2002). Subjective questions to measure welfare and well-being. Recent Findings on Subjective WellBeing. Tinbergen Institute Discussion Paper, TI, 2002-020/3.

Ferrer-I-Carbonell, A. y VAn PraAg, B.M.S. (2006). Insecurity in the labor market: The impact of the type of contract on job satisfaction in Spain and Netherlands. EPICURUS Project Manuscript. Tinbergen Institute. Universidad de Amsterdam.

Frey, B.S. y STUTZER, A. (2002a). "What can economists learn from happiness research?». Journal of Economic Literature, XL (junio), 402-435.

- (2002b). Happiness and economics. Princeton: Princeton University Press.

Gerdtham, U. y Johannesson, M. (2001). «The relationship between happiness, health, and socio-economic factors: Results based on Swedish Microdata». Journal of Socio-Economics, 30, 553-557.

GlaDys MathieU, M. (2005). «Apuntes para el abordaje de la cultura política de los jóvenes: propuesta teórico metodológica». Actas del III SOPCOM, IV LUSOCON y II IBERICO, 509-516. Universidad de Beira Interior.

Gladys Mathieu, M. y Pinto Arboleda, M.C. (2010). "Tratamiento informativo de los jóvenes españoles: Un análisis medioacional de noticias». Question, 28.

Graham, C. (2005). Globalization, poverty, inequality, and Insecurity. Documento de investigación, 2005/33. United Nations University.

Haring-Hidore, M.; STOCK, W.A.; Okun, M.A. y WitTer, R.A. (1985). «Marital status and subjective well-being: A research synthesis». Journal of Marriage and the Family, 47, 947-953. 
Helliwell, J.F. y Putnam, R. (2004). «The social context of well-being». The Royal Society, 1435-1446.

Iglesias de Ussel, J.; Marí Klose, P.; Marí Klose, M. y González Blasco, P. (2010). «Matrimonios y parejas jóvenes. España 2009». Cuadernos Fundación SM, 16.

IngleHart, R. (1990). Culture shifts in advanced industrial society. Princeton: Princenton University Press.

Kahneman, D.; Diener, E. y SCHWarz, N. (1999). Foundations of hedonic psychology: scientific perspectives on enjoyment and suffering. Nueva York: Russell Sage Foundation.

Kahneman, D. y Krueger, A.B. (2006). «Developments in the measurement of subjective well-being». Journal of Economic Perspectives, 20 (1), 3-24.

Layard, R. (2005). Happiness: Lessons from a New Science. Nueva York: Penguin.

LOEWESTEIN, G. (1987). "Anticipation and the valuation of delayed consumption». The Economic Journal, 97, 666-684.

LUCAS, R.E. (2007). «Adaptation and the set-point model of subjective well-being: Does happiness change after major life events?». Current Directions in Psychological Science, 16 (2), 75-79.

Lucas, R.; Clark, A.; Georgellis, Y. y Diener, E. (2004). "Unemployment alters the set points of life satisfaction». Physiological Science, 15 (1), 8-13.

Mateo Rivas, M.J. y Navarro López, M. (1993). Informe de la juventud en España 1992. Madrid: Instituto de la Juventud.

MYers, D.G. (1992). The pursuit of happiness. Nueva York: Willian Morrow.

Navarro López, M. y Mateo Rivas, M.J. (1993). Juventud en cifras 1992. Madrid: Instituto de la Juventud.

Oswald, A.J. (1997). «Happiness and economic performance». Economic Journal, $107,1815-1831$.

ROJAS, M. (2005). "Heterogeneity in the relationship between income and happiness: A conceptual-referent-theory explanation». Journal of Economic Psychology, noviembre.

SÁEz Marín, J. (1995). «Los estudios de la juventud en España: Contextos de un proceso de investigación-acción (1960-1990)». Revista Internacional de Sociología, $10,159-197$.

SANDOVAl ManRíQuez, M. (2007). «Sociología de los valores y la juventud». Última Década, 27, 95-118.

SELIGMAN, M.E.P. (2005). La auténtica felicidad. Barcelona: Byblos.

SENIK, C. (2008). «Is man doomed to progress?». Journal of Economic Behavior \& Organization, 68 (1), 140-152.

THeodossiou, I. (1998). «The effects of low-pay and unemployment on psychological well-being: A logistic regression approach». Journal of Health Economics, 17, 85-104.

Urraco Solamilla, M. (2007). «La sociología de la juventud revisitada: De discursos, estudios, e "historias" sobre los jóvenes». Revista Sociológica de Pensamiento Crítico, 1 (2), 105-126.

VAn PraAg, B. y Ferrer-I-Carbonell, A. (2004). Happiness quantified: A satisfaction calculus approach. Oxford: Oxford University Press.

VeEnHoven, R. (1991). «Is happiness relative?». Social Indicators Research, 24, 1-34.

Winkelman, L. y Winkelman, R. (1998). "Why are the unemployed so unhappy? Evidence from panel data». Economica, 65 (257), 1-15. 


\section{Anexo A. Las medias de muestra}

\begin{tabular}{|c|c|c|}
\hline $\begin{array}{c}\text { Variables } \\
\mathrm{N}^{\circ} \text { observaciones }\end{array}$ & $\begin{array}{c}\text { Media } \\
5.127\end{array}$ & Desviación \\
\hline \multicolumn{3}{|l|}{ Variable dependiente: } \\
\hline Satisfacción & 3,022 & 0,581 \\
\hline \multicolumn{3}{|c|}{ Variables demográficas y socioeconómicas: } \\
\hline Mujer & 0,489 & 0,500 \\
\hline Edad & 22,314 & 4,279 \\
\hline \multicolumn{3}{|l|}{ Estado civil } \\
\hline Casado & 0,111 & 0,314 \\
\hline Con pareja & 0,065 & 0,246 \\
\hline \multicolumn{3}{|l|}{ Actividad principal } \\
\hline Estudia & 0,331 & 0,471 \\
\hline Trabaja & 0,392 & 0,488 \\
\hline Trabaja y estudia & 0,126 & 0,332 \\
\hline Parado & 0,122 & 0,327 \\
\hline Labores del hogar & 0,024 & 0,154 \\
\hline Otra situación & 0,005 & 0,072 \\
\hline \multicolumn{3}{|l|}{ Situación económica } \\
\hline Muy buena & 0,020 & 0,141 \\
\hline Buena & 0,441 & 0,497 \\
\hline Regular & 0,427 & 0,495 \\
\hline Mala & 0,089 & 0,284 \\
\hline Muy mala & 0,023 & 0,151 \\
\hline \multicolumn{3}{|l|}{ Religión } \\
\hline No creyente & 0,316 & 0,465 \\
\hline Creyente & 0,477 & 0,500 \\
\hline Practicante & 0,207 & 0,405 \\
\hline \multicolumn{3}{|l|}{ Variables particulares: } \\
\hline \multicolumn{3}{|c|}{ Expectativa económica para el próximo año } \\
\hline Mejor & 0,292 & 0,455 \\
\hline Igual & 0,498 & 0,500 \\
\hline Peor & 0,195 & 0,396 \\
\hline \multicolumn{3}{|l|}{ Tipo de escuela } \\
\hline Publica & 0,742 & 0,437 \\
\hline Religiosa & 0,183 & 0,387 \\
\hline Privada & 0,075 & 0,263 \\
\hline \multicolumn{3}{|l|}{ Tipo de contrato (si trabaja) } \\
\hline Contrato permanente & 0,34 & 0,48 \\
\hline Contrato temporal & 0,55 & 0,50 \\
\hline Empresario & 0,01 & 0,12 \\
\hline Autónomo & 0,08 & 0,27 \\
\hline Otros & 0,01 & 0,11 \\
\hline \multicolumn{3}{|l|}{ Convivencia } \\
\hline Vive con padres & 0,727 & 0,446 \\
\hline Vive independiente & 0,211 & 0,408 \\
\hline Comparte vivienda & 0,061 & 0,239 \\
\hline Otra situación & 0,002 & 0,044 \\
\hline
\end{tabular}

Fuente: elaboración propia. 